\title{
Beractant and poractant alfa in premature neonates with respiratory distress syndrome: a systematic review of real-world evidence studies and randomized controlled trials
}

\author{
Manuel Sánchez Luna $\mathbb{C}^{1} \cdot$ Peter Bacher $^{2} \cdot$ Kristina Unnebrink $^{3} \cdot$ Marisol Martinez-Tristani $^{2} \cdot$ Cristina Ramos Navarro $^{1}$
}

Received: 23 August 2019 / Revised: 16 December 2019 / Accepted: 31 January 2020 / Published online: 12 February 2020

(c) The Author(s) 2020. This article is published with open access

\begin{abstract}
Findings from previous meta-analyses of randomized clinical trials (RCTs) in premature infants with respiratory distress syndrome (RDS) varied as to whether clinical outcomes differed by type of animal-derived pulmonary surfactant; real-world evidence (RWE) was excluded. We extracted study characteristics and outcomes from full-text articles from a systematic search for studies that compared beractant with poractant alfa for RDS in preterm infants. RWE data were tabulated; RCT data were subjected to meta-analyses. Designs, patient characteristics, and follow-up durations varied widely among studies (4 RWE, 15 RCT). RWE studies with adjusted odds ratios (ORs) found no statistically significant between-treatment differences in outcomes. In RCT meta-analyses, no statistically significant between-treatment differences were observed for death (OR [95\% confidence interval], 1.35 [0.98-1.86]), bronchopulmonary dysplasia (1.25 [0.96-1.62]), pneumothorax (1.21 [0.72-2.05]), and air leak syndrome (2.28 [0.82-6.39]). Collectively, outcomes were similar with beractant and poractant alfa in RWE studies and pooled RCTs.
\end{abstract}

\section{Introduction}

Respiratory distress syndrome (RDS) is common in premature neonates, occurring in $\sim 80 \%$ of those born at or $<28$ weeks gestational age (wGA) [1], and is usually caused by inadequate levels of endogenous lung surfactant [2]. When supportive measures, such as continuous positive airway pressure, are insufficient to maintain oxygenation in preterm infants with RDS, the introduction of exogenous

Supplementary information The online version of this article (https:// doi.org/10.1038/s41372-020-0603-7) contains supplementary material, which is available to authorized users.

Manuel Sánchez Luna

msluna@salud.madrid.org

1 Division of Neonatology, Instituto de Investigación Sanitaria Gregorio Marañón, Complutense University of Madrid, Hospital General Universitario “Gregorio Marañón”, O’Donnell 48, 28009 Madrid, Spain

2 Global Medical Affairs, Pharmaceutical Research and Development, AbbVie Inc., 1 North Waukegan Road, North Chicago, IL 60064, USA

3 Data and Statistical Sciences, AbbVie Deutschland GmbH \& Co. KG, Knollstraße, 67061 Ludwigshafen, Germany surfactant into the trachea as rescue therapy is an important means of reducing the risk of serious complications and adverse outcomes, such as death, bronchopulmonary dysplasia (BPD), and air leak syndrome (ALS) [1, 3]. Guidelines state that natural (animal-derived) surfactants are preferred over synthetic surfactants because the former are associated with lower rates of adverse outcomes [1, 3].

The two most widely used animal-derived surfactants are beractant and poractant alfa [4]. Beractant is an extract from bovine lungs and contains $25 \mathrm{mg} / \mathrm{mL}$ phospholipids, whereas poractant alfa is an extract from porcine lungs and contains 74-76 mg/mL phospholipids; both preparations contain smaller concentrations of natural surfactant-associated proteins and are suspended in $0.9 \%$ saline solution [5-8]. Beractant and poractant alfa as rescue therapy for RDS in preterm infants were compared in previous meta-analyses [9, 10]. One metaanalysis found significantly greater rates of death before hospital discharge and of several other endpoints (composite outcome of death or BPD, defined as need for oxygen at 36 weeks postmenstrual age [PMA]; multiple surfactant dosing; and patent ductus arteriosus requiring treatment with a cyclo-oxygenase inhibitor) with beractant compared with poractant alfa; however, no significant difference was found between the two treatments for death before 28 days of age or any other endpoint (BPD at 28-30 days of age, BPD at 
36 weeks PMA, pneumothorax, ALS, pulmonary hemorrhage, confirmed bacterial sepsis, necrotizing enterocolitis, periventricular leukomalacia, retinopathy of prematurity, retinopathy of prematurity stage $\geq 3$, intraventricular hemorrhage, and intraventricular hemorrhage stage $\geq 3$ ) [9]. The other metaanalysis, which focused on mortality, found no significant difference between beractant and poractant alfa for the rate of death [10]. However, neither of these previous meta-analyses aimed to describe real-world evidence (RWE) studies, which might provide valuable additional information on the actual implementation of therapies [11] and are of interest to regulatory authorities [12].

The objective of this analysis was to systematically compare up-to-date published results from peer-reviewed journal articles, both from RWE studies and RCTs, of beractant versus poractant alfa for the rescue treatment of RDS in premature neonates.

\section{Material and methods}

\section{Literature search}

The systematic literature search included the following search terms: (beractant or Survanta) and (poractant or Curosurf) and (RDS) and (comparative study or "vs." or "versus" or compar* or "head to head") and (prematur* or preterm or infant or newborn or baby or babies or neonate or birth or childbirth or preemie or $<37$ weeks [comprising $<29,<30,<31,<32,<33,<34,<35,<36$, and <37 weeks]). The BIOSIS Previews ${ }^{\circledR}$, Current Contents ${ }^{\circledR}$ Search, Derwent Drug File, Embase ${ }^{\circledR}$ EMCare ${ }^{\circledR}$, International Pharmaceutical Abstracts, MEDLINE ${ }^{\circledast}$, and SciSearch ${ }^{\circledR}$ databases were searched on June 28, 2018; an updated search was conducted on March 27, 2019, but found no additional studies. No limits were applied for publication dates or language.

\section{Literature selection}

Based on abstracts from the literature search, articles about prospective or retrospective clinical studies that compared beractant and poractant alfa for the treatment (but not prevention) of RDS in premature infants were selected. Duplicates, meeting abstracts, review articles, prior metaanalyses, and other inapplicable sources were removed. Full-text versions of the remaining articles were reviewed and those not meeting the study inclusion criteria or not including the endpoints of interest were discarded.

\section{Data extraction}

Data were extracted only from the remaining full-text articles, not from abstracts. When available, the following information was extracted from each article: study design characteristics (e.g., use of blinding, duration of follow-up), key patient eligibility criteria (e.g., gestational age, place of birth, birth weight limit, age at randomization or first dose, level of respiratory support and oxygen parameters, exclusion because of comorbidities), the number of patients in each dose group, patient characteristics (gestational age, weight, cesarean delivery, sex, exposure to prenatal steroids, fractional inspired oxygen $\left[\mathrm{FiO}_{2}\right]$, and the number of times that surfactant was actually administered), definitions of extracted endpoints, and the incidence of endpoints of interest (death, BPD, pneumothorax, and ALS). Study quality was assessed using published grading schemes [9, 13, 14]. The composite endpoint of BPD or death was extracted only for the RWE studies. For the RCTs, the composite endpoint was not extracted because few RCTs reported such data. For RWE studies only, the published odds ratios (ORs), confidence intervals (CIs), and $P$ values were extracted to be presented in the current article. Due to the heterogeneity in study designs and adjusted analyses, no meta-analysis was performed on published RWE studies. Results from RWE studies are reported and discussed separately in the current article. If there was ambiguity during data extraction, the authors of the articles were contacted for guidance with proper understanding of their studies.

\section{Meta-analyses}

The primary meta-analyses of RCTs were conducted with all dosing regimens for beractant or poractant alfa aggregated. For trials with multiple dosing arms per product, the respective arms were pooled. A sensitivity analysis included only RCTs or individual RCT treatment arms in which the dosage was consistent with the US product label. The product label for beractant recommends a dose of $4 \mathrm{~mL} / \mathrm{kg}$ of patient body weight, as often as every $6 \mathrm{~h}$; because the concentration of the surfactant suspension is $25 \mathrm{mg} / \mathrm{mL}$, this corresponds to $100 \mathrm{mg} / \mathrm{kg}$ surfactant $[5,7]$. The US product label for poractant alfa recommends an initial dose of $2.5 \mathrm{~mL} / \mathrm{kg}$, followed by $1.25 \mathrm{~mL} / \mathrm{kg}$, which may be administered at $\sim 12$-h intervals; because the concentration of the surfactant suspension is $80 \mathrm{mg} / \mathrm{mL}$, this corresponds to $200 \mathrm{mg} / \mathrm{kg}$ followed by $100 \mathrm{mg} / \mathrm{kg}$ surfactant [6]. Another sensitivity analysis compared equal weight-based doses of beractant and poractant alfa $(100 \mathrm{mg} / \mathrm{kg}$ each). Finally, sensitivity analyses were conducted to compare the incidence of BPD defined as the need for oxygen at 28 days after birth or as assessed at 36 weeks postconceptional age (PCA) or PMA. The metaanalyses were conducted with a random-effects model using the Mantel-Haenszel method. A random-effects approach was used because of the inherent heterogeneity 


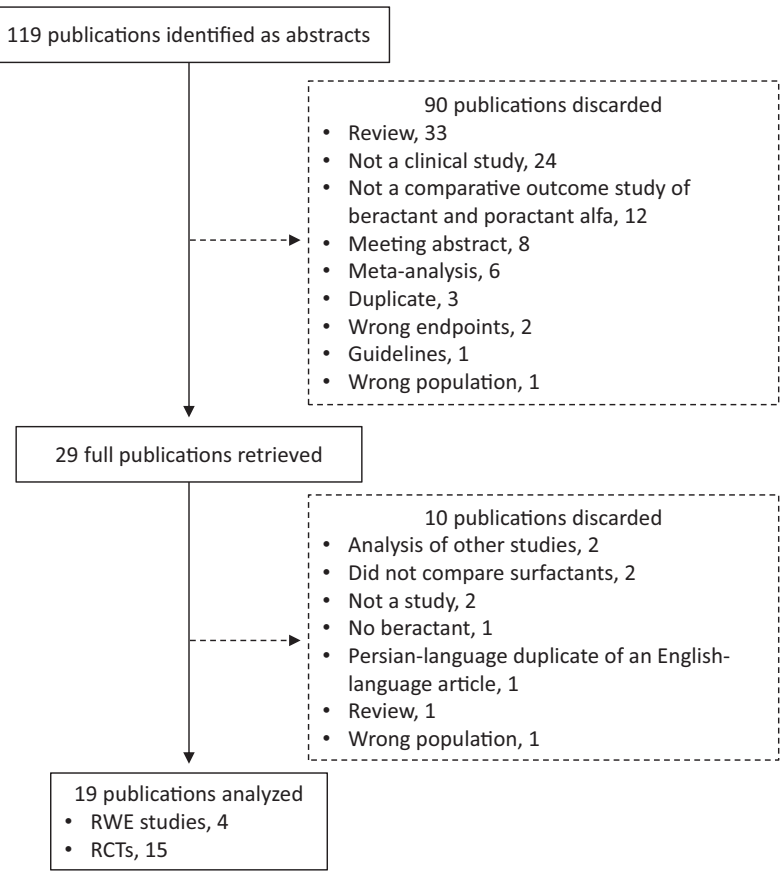

Fig. 1 Literature search results. RCT randomized controlled trial, RWE real-world evidence.

between the RCTs driven, for example, by different study durations. For the RCTs, the meta-analyses provided ORs, 95\% CIs, and $P$ values. In some particular cases, risk differences with $95 \%$ CIs and $P$ values are also reported. All meta-analyses were conducted using Review Manager version 5.3 (Copenhagen: The Nordic Cochrane Centre, The Cochrane Collaboration, 2014).

\section{Results}

\section{Literature selection}

The systematic literature search yielded 119 abstracts, of which 90 were discarded (Fig. 1). Twenty-nine full-text articles were retrieved, of which 10 were eliminated. The remaining 19 articles (4 RWE studies [15-18] and 15 RCTs [19-33]) were included.

\section{RWE studies}

\section{Study and patient characteristics}

Four RWE studies were analyzed (Table 1) [15-18]. Two studies had mid- to long-term outcomes $(>72 \mathrm{~h})[15,17]$, and two studies did not define specific minimum times for patient follow-up [16, 18]. The criterion for gestational age was $<37$ wGA in two studies $[15,18]<32$ wGA in 1 study [17], and 25-32 wGA in another study [16]. Only 1 study had a limit (500-1999 g) for birth weight [16]. The dosages were according to the US product label (i.e., beractant $100 \mathrm{mg} / \mathrm{kg}$; poractant alfa $200 \mathrm{mg} / \mathrm{kg}$ for the first dose; and $100 \mathrm{mg} / \mathrm{kg}$ for subsequent doses) in 1 study [17], beractant $100 \mathrm{mg} / \mathrm{kg}$ and poractant alfa $100 \mathrm{mg} / \mathrm{kg}$ for all doses in another study [18], and unspecified in two studies [15, 16]. Although not explicitly stated, it is expected that in the RWE studies, approved doses were used. Two studies included large numbers of patients $(51,282$ and 14,173) $[15,16]$, whereas the other two studies were of more modest sizes (415 and 242) [17, 18]. Median or mean gestational age ranged from $\sim 27$ to 30 weeks. Median or mean birth weight ranged from 1037 to $2026 \mathrm{~g}$ among studies, trending higher with greater gestational age. The proportions of patients who were delivered by cesarean section ranged from $56.1 \%$ to $82.6 \%$. The proportion of male patients ranged from $51.6 \%$ to $64.0 \%$. The use of prenatal steroids ranged from high to nearly universal (range, 63.7-95.9\%). Baseline values for $\mathrm{FiO}_{2}$ were not reported. One study reported data on repeat dosing, which was comparable between the two treatment groups (mean number of doses: beractant, 1.78; poractant alfa, 1.63) [17]. Quality assessment of RWE studies is presented in Supplementary Table 1.

\section{Patient outcomes}

Deaths were reported in all four RWE studies (Table 1; Fig. 2). The incidence of death was similar between the beractant and poractant alfa groups in three studies [15-17] but significantly lower with beractant versus poractant alfa in 1 study (2/74 [2.7\%] vs. $19 / 168[11.3 \%] ; P=0.027)$, which was also the only study that specifically compared a $100-\mathrm{mg} / \mathrm{kg}$ dose for each surfactant [18]; a $100 \mathrm{mg} / \mathrm{kg}$ dose of poractant alfa is not the currently approved dosage [6]. The incidence of BPD was reported in two studies $[15,17]$ and was similar in the beractant and poractant alfa groups in the 1 study that tested for a significant difference between treatments for this outcome [17]. The composite endpoint of BPD or death occurred with similar incidence in the beractant and poractant alfa groups in the two studies that reported this outcome $[15,17]$. Pneumothorax was reported in 1 study, but there was no statistical test for that outcome [15]. The incidence of ALS was reported in two studies and was similar in the beractant and poractant alfa groups in both reports $[15,17]$.

\section{Randomized controlled trials}

\section{Study and patient characteristics}

Fifteen RCTs were analyzed (Table 2) [19-33]. The followup times for study outcomes were generally not explicitly 


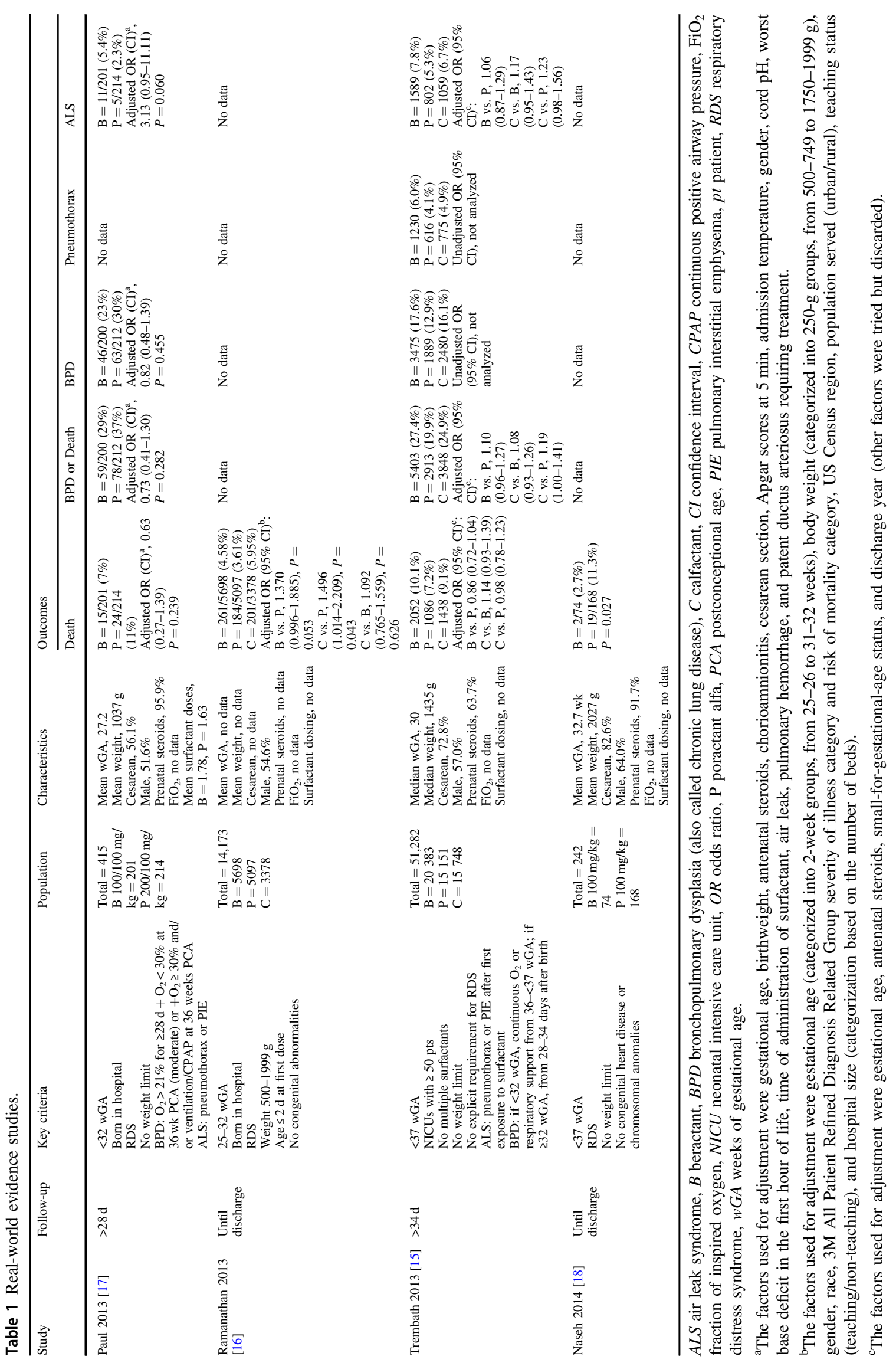




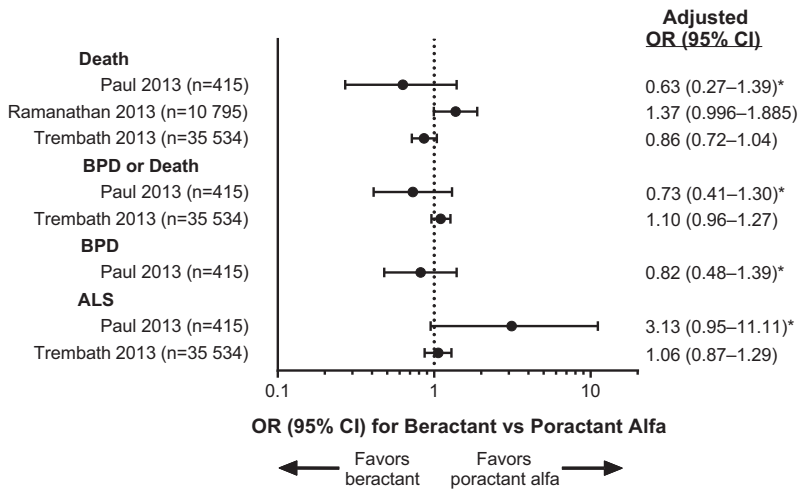

Fig. 2 Forest plot of patient outcomes from RWE studies that reported adjusted analyses. The numbers of patients who received beractant or poractant alfa (but not other surfactants) are shown. The ORs and $95 \%$ CIs are given based on the values from the original articles, which had been adjusted for patient characteristics and other factors. Note: only 1 study specifically compared a $100-\mathrm{mg} / \mathrm{kg}$ dose for each surfactant [18]. ALS air leak syndrome, BPD bronchopulmonary dysplasia, CI confidence interval, OR odds ratio, RWE real-world evidence. *The factors used for adjustment were gestational age, birthweight, antenatal steroids, chorioamnionitis, cesarean section, Apgar scores at $5 \mathrm{~min}$, admission temperature, gender, cord $\mathrm{pH}$, worst base deficit in the first hour of life, time of administration of surfactant, air leak, pulmonary hemorrhage, and patent ductus arteriosus requiring treatment. The factors used for adjustment were gestational age (categorized into 2-week groups, from 25-26 to 31-32 weeks), body weight (categorized into 250-g groups, from 500-749 to 1750-1999 g), gender, race, 3M All Patient Refined Diagnosis Related Group severity of illness category and risk of mortality category, US Census region, population served (urban/rural), teaching status (teaching/nonteaching), and hospital size (categorization based on the number of beds). ${ }^{\ddagger}$ The factors used for adjustment were gestational age, antenatal steroids, small-for-gestational-age status, and discharge year (other factors were tried but discarded).

defined but appeared to range from $72 \mathrm{~h}$ to $\geq 28$ days or $>36$ weeks PCA for some endpoints. Fourteen studies had mid-term to long-term outcomes ( $>72 \mathrm{~h})[19,20,22-33]$, and 1 study had short-term outcomes $(\leq 72 \mathrm{~h})$ [21]. The criterion for gestational age was $<37$ or $\leq 37$ wGA in five studies $[22,24,25,29,32],<35 \mathrm{wGA}$ in two studies $[19,26], \leq 32 \mathrm{wGA}$ in three studies [20, 27, 28], <30 wGA in one study [31], 26-36 wGA in one study [33], and 24 to $<30$ wGA in one study [21]. Two studies simply stated that patients had to be preterm, without defining a specific gestational age [23, 30]. Four studies imposed a limit on birth weight for enrollment: $700-1500 \mathrm{~g}$ [30], 750-1750 g [19], $>750 \mathrm{~g}$ [26], and $\leq 2000 \mathrm{~g}$ [28]. The studies included between 30 and 293 patients. Nine studies included data about doses that were administered according to the US product label (i.e., beractant $100 \mathrm{mg} / \mathrm{kg}$; poractant alfa $200 \mathrm{mg} / \mathrm{kg}$ for the first dose, and $100 \mathrm{mg} / \mathrm{kg}$ for subsequent doses) [19, 20, 22, 23, 25, 27, 29, 31, 33]. Six studies specifically compared beractant $100 \mathrm{mg} / \mathrm{kg}$ with poractant alfa $100 \mathrm{mg} / \mathrm{kg}[19,24,26,28,30,32]$. Median or mean gestational age ranged from $\sim 26$ to 33 weeks. Median or mean birth weight ranged widely among studies, from 731 to $1911 \mathrm{~g}$, trending higher with greater gestational age. The proportions of patients who were delivered by Cesarean section ranged from $57.6 \%$ to $83.0 \%$. The proportion of male patients ranged from $41.3 \%$ to $66.7 \%$. The use of prenatal steroids ranged from low to nearly universal (range, 30.0-98.1\%). Baseline values for $\mathrm{FiO}_{2}$ in the five studies for which data were reported ranged widely, from $\sim 0.5$ to 0.9 $[19,23,28-30]$. Six studies reported the mean number of doses of surfactant (range, 1.06-2.2) [22, 23, 25, 26, 29, 30] and six studies reported the proportion of patients who received $>1$ dose (range, 13-68\%) [19, 21, 24, 27, 28, 31]; by either measure, more doses of beractant than poractant alfa were given in most studies. Three studies had BPD data at 28 weeks after birth [19, 22, 32], and six studies assessed BPD at 36 weeks PCA or PMA [23, 27-31].

Eight RCTs were included in the previous meta-analysis conducted by Singh et al.; we confirmed the grading conferred by the authors of that publication for those eight studies. Grading for the additional seven studies identified in the current meta-analysis is presented in Supplementary Table 2.

\section{Patient outcomes}

Thirteen mid-term to long-term RCTs of beractant and poractant alfa provided data on death [19, 20, 23-33], 12 on BPD [19, 20, 22, 23, 25, 27-33], 9 on pneumothorax [19, 22, 25-27, 29, 30, 32, 33], and 3 on ALS [23, 28, 30]; the short-term RCT, not included in the meta-analyses, provided data on death, BPD, and ALS (Table 2) [21].

Primary meta-analysis results and individual studies demonstrated no significant differences (i.e., 95\% CI of the OR encompassed 1) between treatment with beractant compared with poractant alfa for death, BPD, pneumothorax, and ALS (Fig. 3). The sensitivity analysis that included only data from doses of surfactant that complied with the US product labels ( $n=9$ studies) generally supported the findings of the primary meta-analyses $[19,20,22,23,25,27,29,31,33]$; the comparisons between beractant and poractant alfa were not statistically significant for death, pneumothorax, and ALS (Fig. 4). The incidence of BPD was of borderline significance (overall treatment effect, $P=0.05$ ) overall but not in individual studies (Fig. 4). The risk difference of BPD estimated in the sensitivity analysis was 0.04 (95\% CI: -0.00 to $0.08 ; P=$ $0.07)$. The sensitivity analysis that compared only data about beractant $100 \mathrm{mg} / \mathrm{kg}$ versus poractant alfa $100 \mathrm{mg} / \mathrm{kg}$ ( $n=6$ studies $[19,24,26,28,30,32])$ revealed no significant differences in outcomes in any individual study or the meta-analyses (Fig. 5). In the sensitivity analyses of data for which BPD was defined as the need for oxygen at day 28 after birth $(n=3$ studies $[19,22,32])$ or evaluated at 


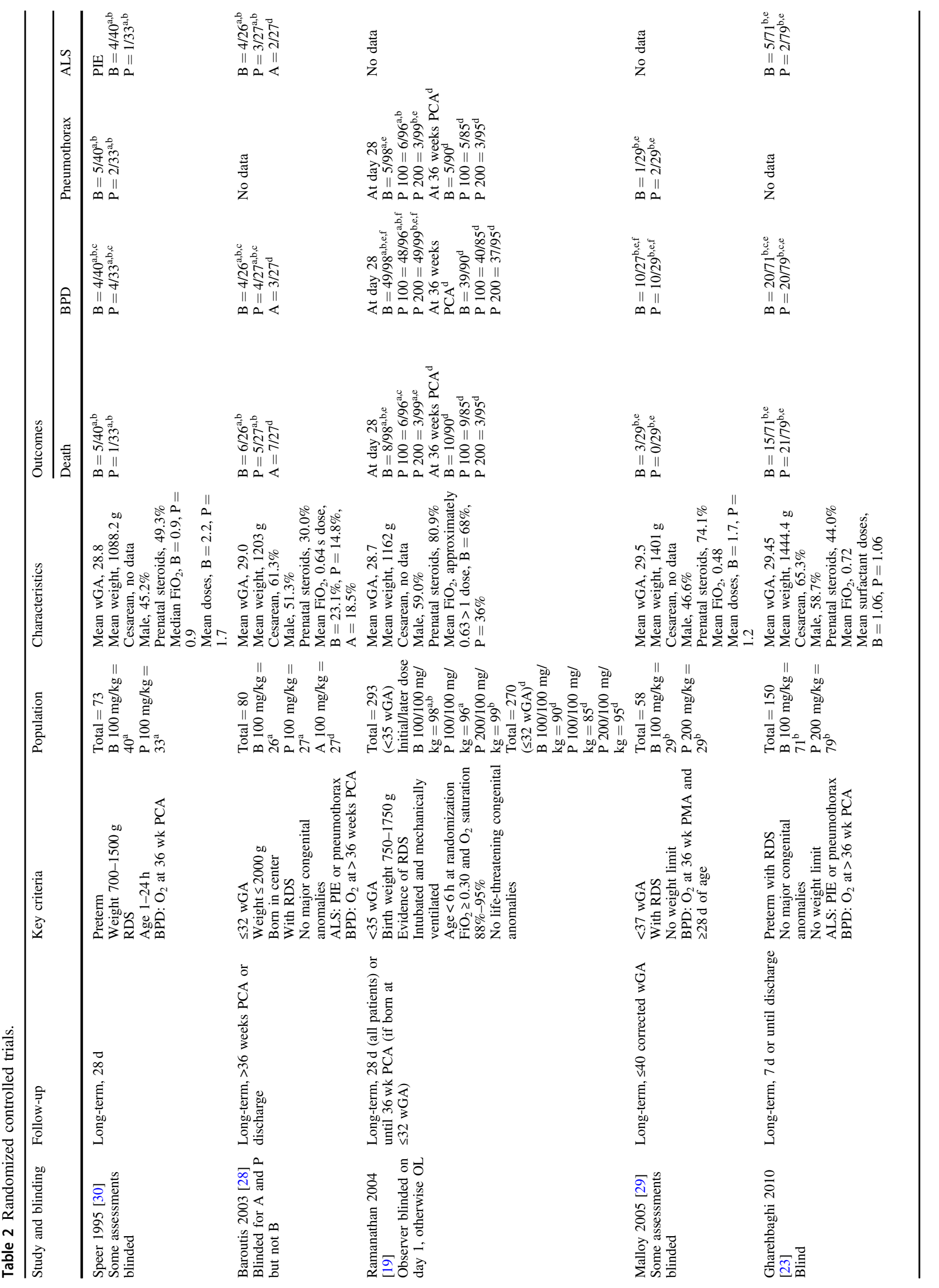




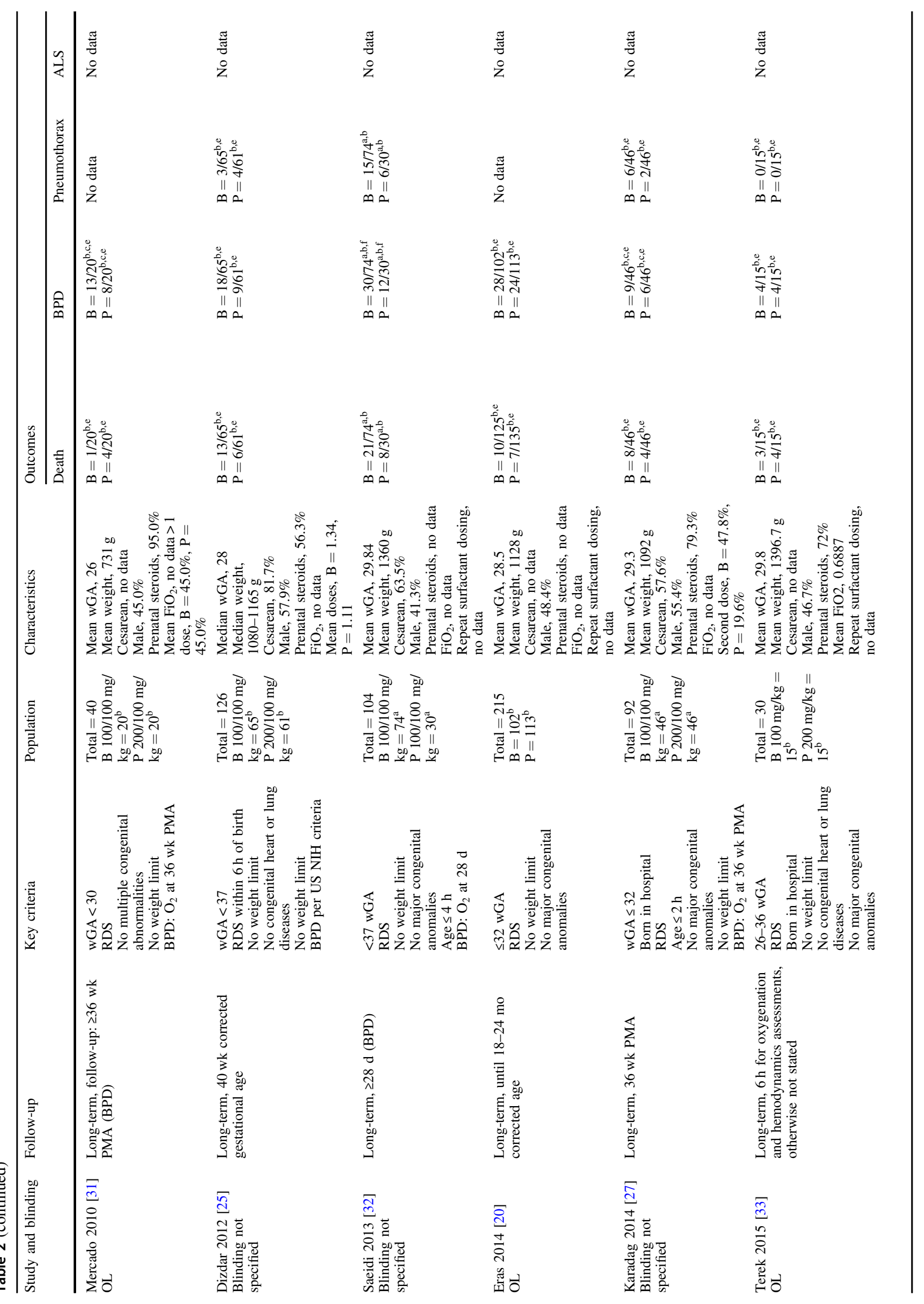




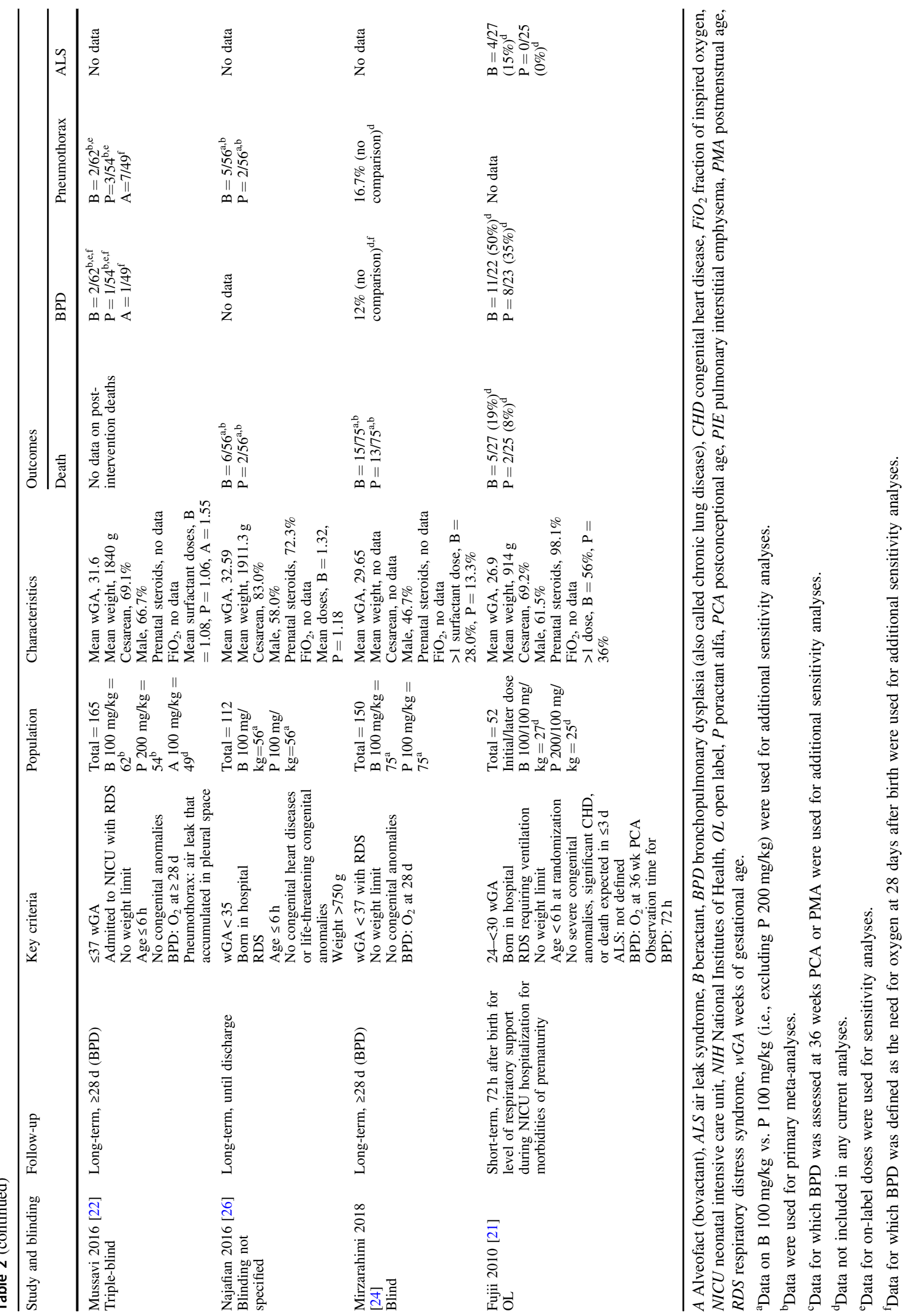



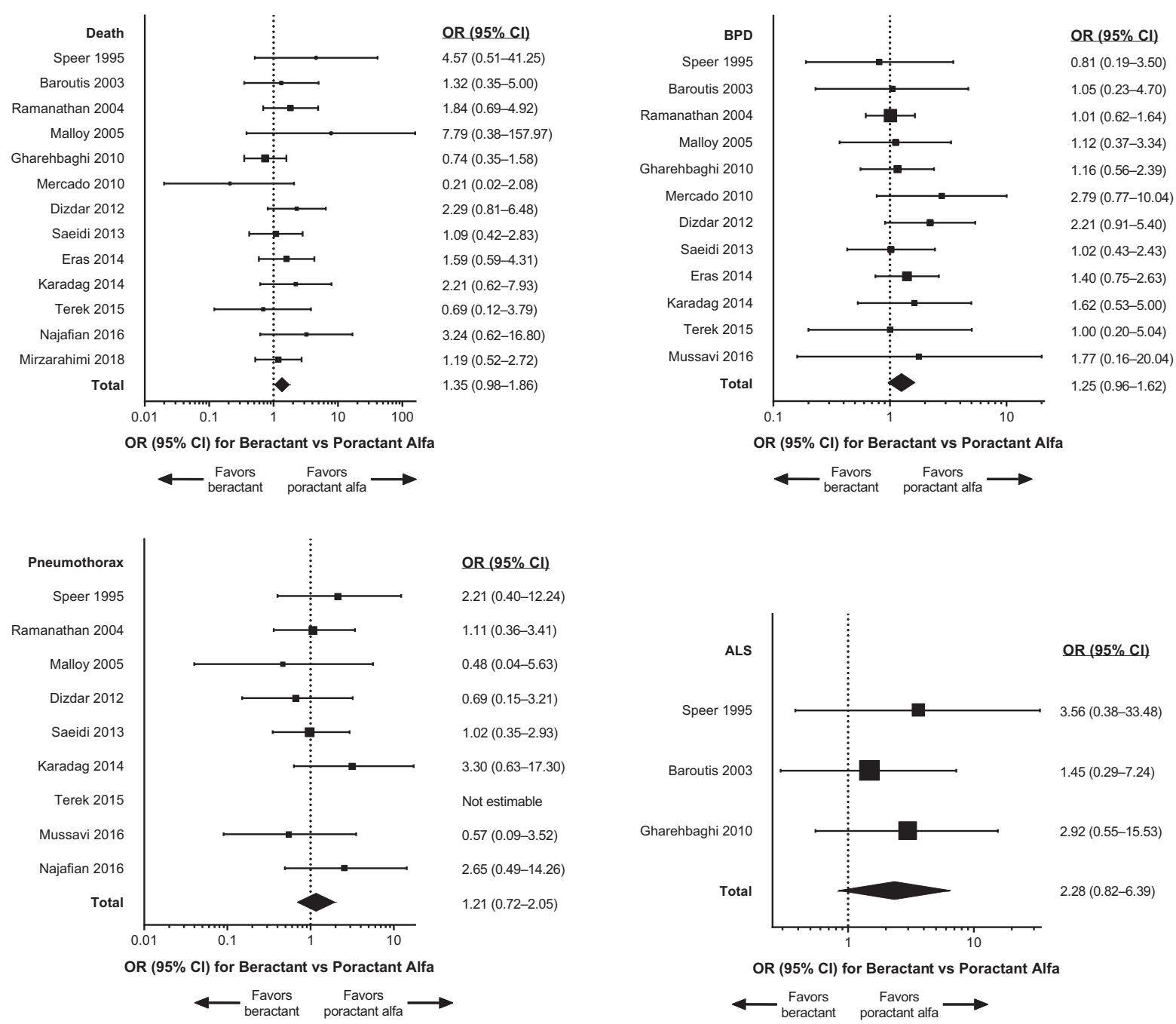

Fig. 3 Forest plots of patient outcomes from primary meta-analyses of RCTs, with any dose of surfactant. ALS air leak syndrome, BPD bronchopulmonary dysplasia, CI confidence interval, OR odds ratio, RCT randomized controlled trial.

36 weeks PCA or PMA ( $n=6$ studies [23, 27-31]), the overall analyses and individual study results did not demonstrate significant differences between beractant and poractant alfa (all doses of beractant and poractant alfa included; Fig. 6).

The original publication of the short-term $(<72 \mathrm{~h}$ of follow up) RCT, which was not included in the meta-analyses, reported no significant differences between treatments for the incidences of death and BPD, whereas there was a trend favoring poractant alfa over beractant for prevention of ALS [21].

\section{Discussion}

The objective of this analysis was to compare clinical outcomes with beractant and poractant alfa using the most current published data from RWE studies and RCTs in premature infants treated for acute RDS. The primary results indicated that outcomes were not statistically significantly different in almost all individual RWE studies and RCTs, and that death, BPD, pneumothorax, and ALS occurred with similar incidence in the meta-analyses of RCTs. The only RWE study that compared the incidence of BPD with beractant and poractant alfa found no significant difference between treatments [17].

RWE studies and RCTs provide complementary information; RWE studies are more representative of real-world clinical practice, whereas RCTs are more rigorously controlled for factors other than the treatment of interest. A full understanding of the relative utility of a therapy is best derived from both kinds of evidence. However, comparison of the results of the RWE studies and the RCTs is complicated by several factors. There was substantial variation among the analyzed studies in design, entry criteria, demographic and disease characteristics, dosing regimens, 


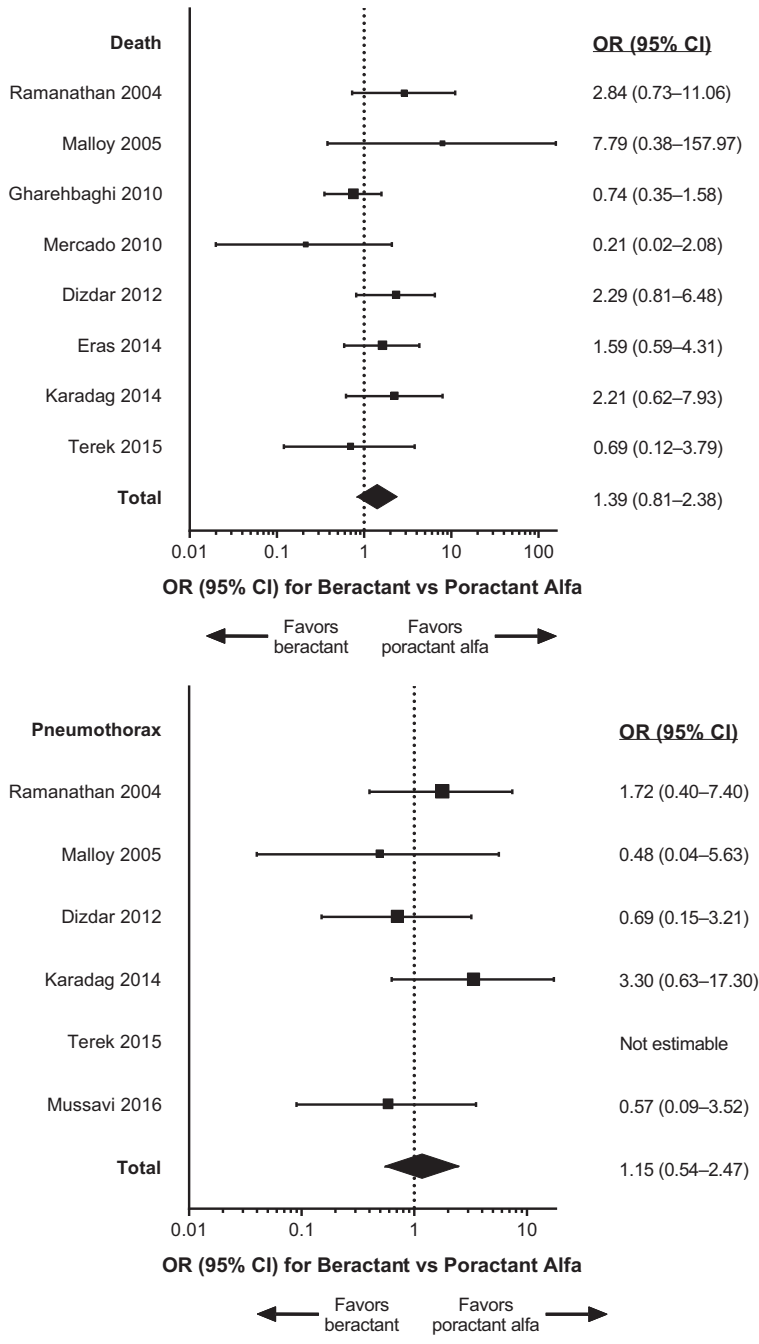

Fig. 4 Forest plots of patient outcomes from sensitivity analysis that included only data from RCTs or RCT treatment arms in which doses of surfactant were given according to the US product

definitions and reporting of endpoints, and follow-up time. The RWE studies were reported during a narrow range of time (2013-2014), whereas the RCTs were reported over a much longer period (1995-2018); thus, it is possible that aspects of therapy, including evolution of care, other than the type of surfactant may have differed. Treatment in the RWE studies was not randomized and was always openlabel, whereas it was sometimes blinded in the RCTs. Finally, the populations of two of the RWE studies were far larger than the populations of any of the RCTs, resulting in much greater power to detect statistical differences between groups; counteracting this was the greater heterogeneity of the RWE populations due to lack of restrictions in inclusion criteria, controlled selection of the population enrolled, and treatment approach, although this was at least partially compensated via adjusted analyses in the original articles. Despite these caveats, the general consistency of the results among RWE studies and the meta-analyses of RCTs
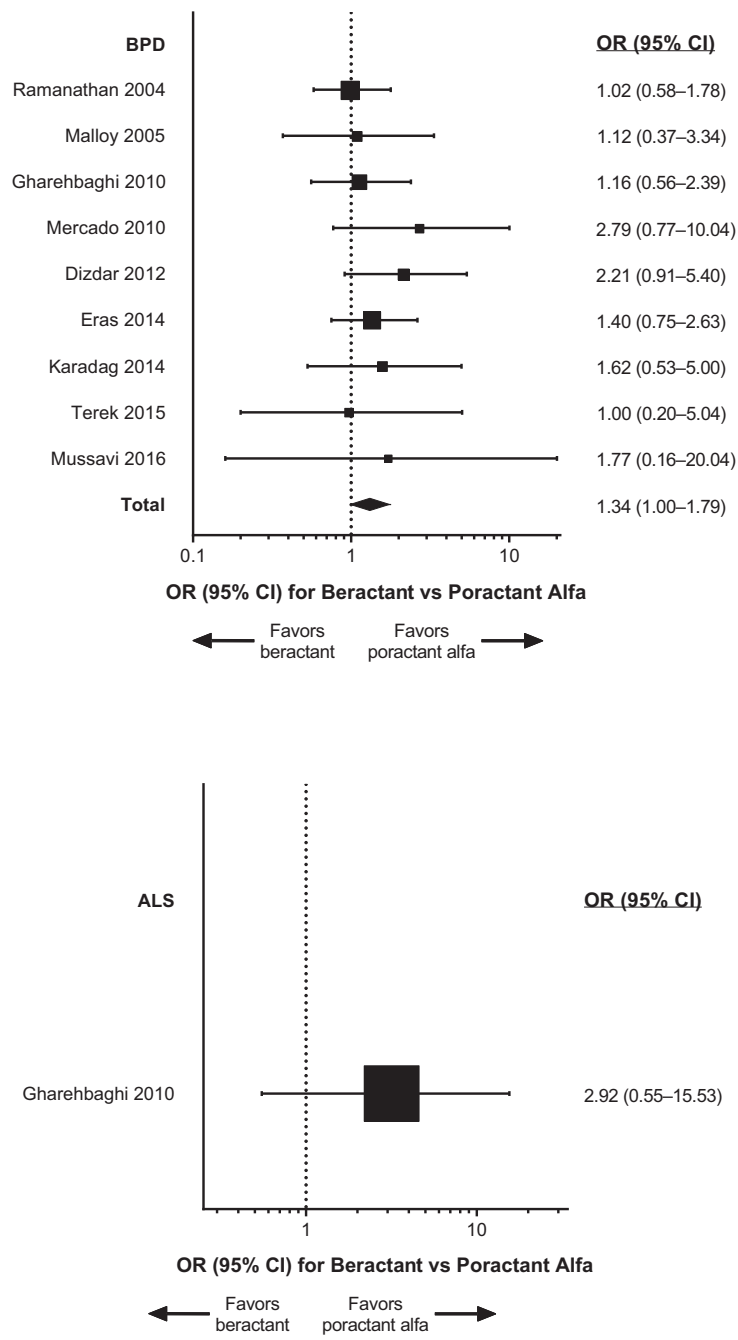

label. ALS air leak syndrome, BPD bronchopulmonary dysplasia, CI confidence interval, OR odds ratio, RCT randomized controlled trial.

suggest that the findings were valid. Further large-scale research could shed more light on whether there is a realworld difference between beractant and poractant alfa treatment for the outcome of BPD. The monitoring of postmarketing safety would provide relevant information to regulatory agencies and would be essential in updating US product labels, as needed. Given the vulnerable population of premature neonates and the criticality of RDS, as well as the evolution of standard of care in the last 20 years, it is relevant and critical to continue to monitor and update healthcare professionals on the safety of these products, particularly in real-world scenarios.

Our RCT meta-analysis results were in agreement with the findings of a previous meta-analysis reported by Singh et al. [9] for several outcomes but differed for the risk of death. We found no significant difference between beractant and poractant alfa in the risk of death, whereas Singh et al. reported an increased risk of death with beractant compared 


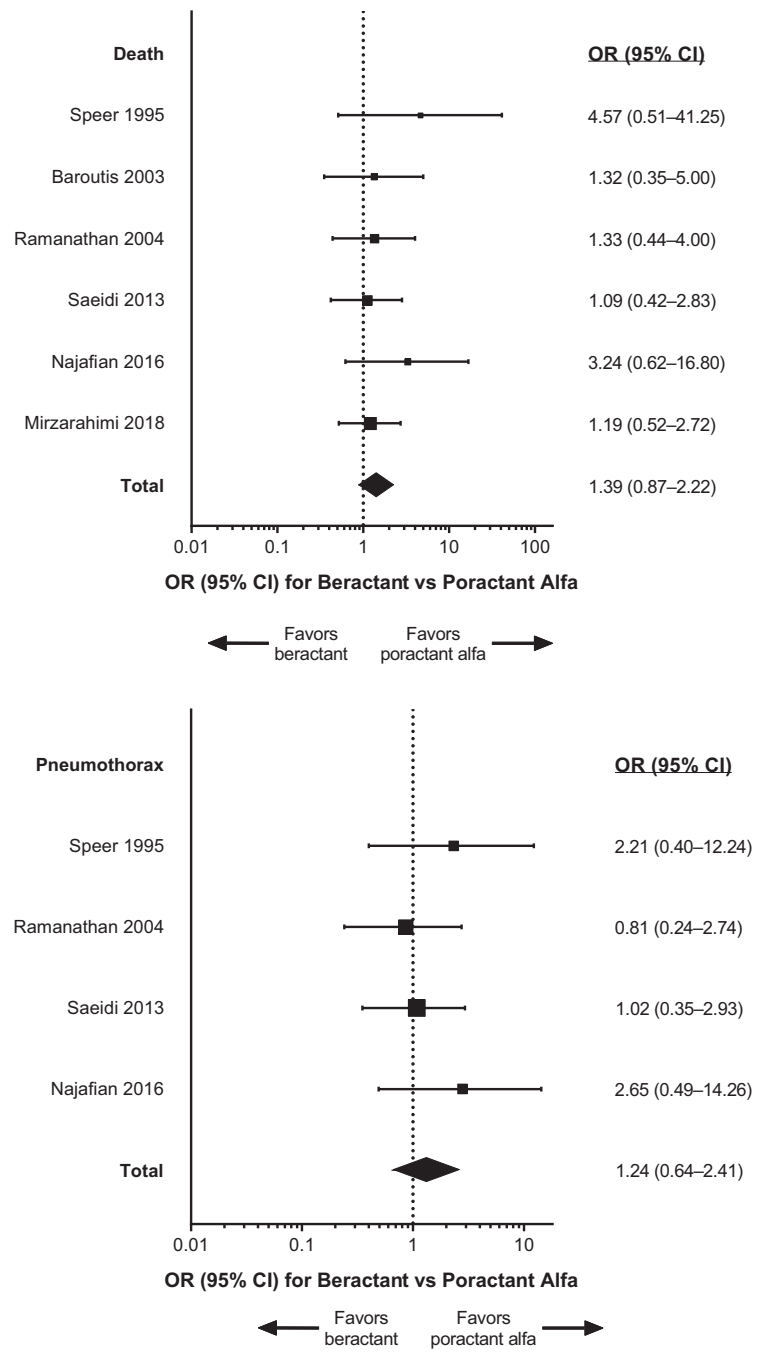

Fig. 5 Forest plots of patient outcomes from sensitivity analysis that included only data from RCTs or RCT treatment arms with $100 \mathrm{mg} / \mathrm{kg}$ doses of surfactant. ALS air leak syndrome, BPD

with poractant alfa (risk ratio [95\% CI], 1.44 [1.04-2.00]) [9]. However, the difference in mortality reported by Singh et al. was observed only for higher doses of poractant alfa, whereas our own findings were consistent regardless of dosing regimen. Additionally, the network meta-analysis of Zhang et al., which included only death as an outcome, agreed with our results, finding no significant difference in mortality between treatment with beractant and poractant alfa (OR [95\% CI], 1.27 [0.99-1.62]) [10]. Our primary finding for BPD and sensitivity analyses based on specific definitions were comparable to the conclusion of Singh et al. that there was a similar risk of BPD with either treatment according to two different definitions of BPD (need for oxygen at 28-30 days old, risk ratio [95\% CI], 0.97 [0.77-1.23]; need for oxygen at 36 weeks PMA, 0.94 [0.79-1.12]). Our results agree with those of Singh et al. for the risks of pneumothorax and ALS in that there was no
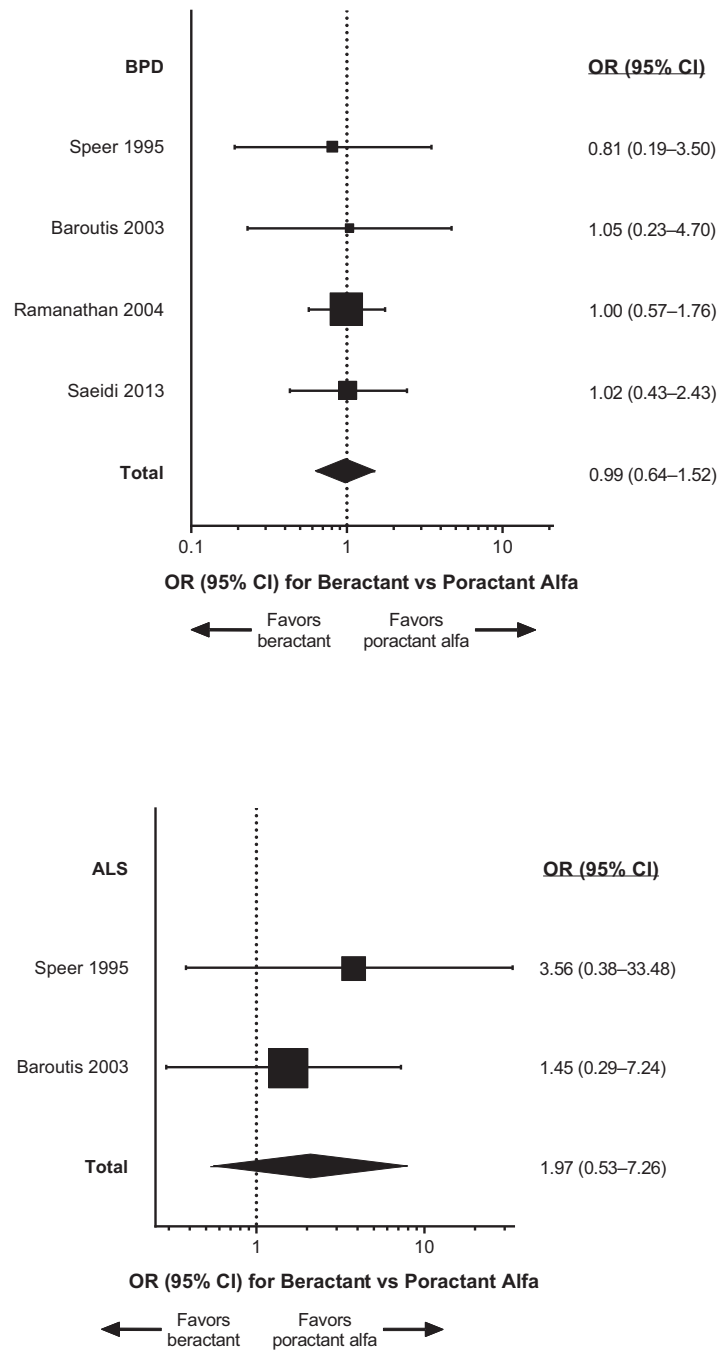

bronchopulmonary dysplasia, CI confidence interval, OR odds ratio, $\mathrm{RCT}$ randomized controlled trial.

significant difference between beractant and poractant alfa. A recent meta-analysis published by Tridente et al. in 2019 [34] concluded that poractant alfa was significantly more effective than bovine-derived surfactants at prevention of BPD in preterm infants with RDS, but that finding is not comparable to our work because it was based on combined data from beractant and two other bovine-derived surfactants (bovactant and bovine lipid extract surfactant suspension) that may have nonequivalent properties.

Among the main factors that could have led to differences in conclusions among meta-analyses are the number and types of studies that were pooled. We included some of the same studies as the previous meta-analyses of Singh et al. and Zhang et al. [9, 10]. However, we also added 9 studies that were not represented in either of the earlier meta-analyses; these included 2 RWE studies $[17,18]$ and 7 RCTs $[20,22,24,26,31-33]$. The seven additional RCTs 


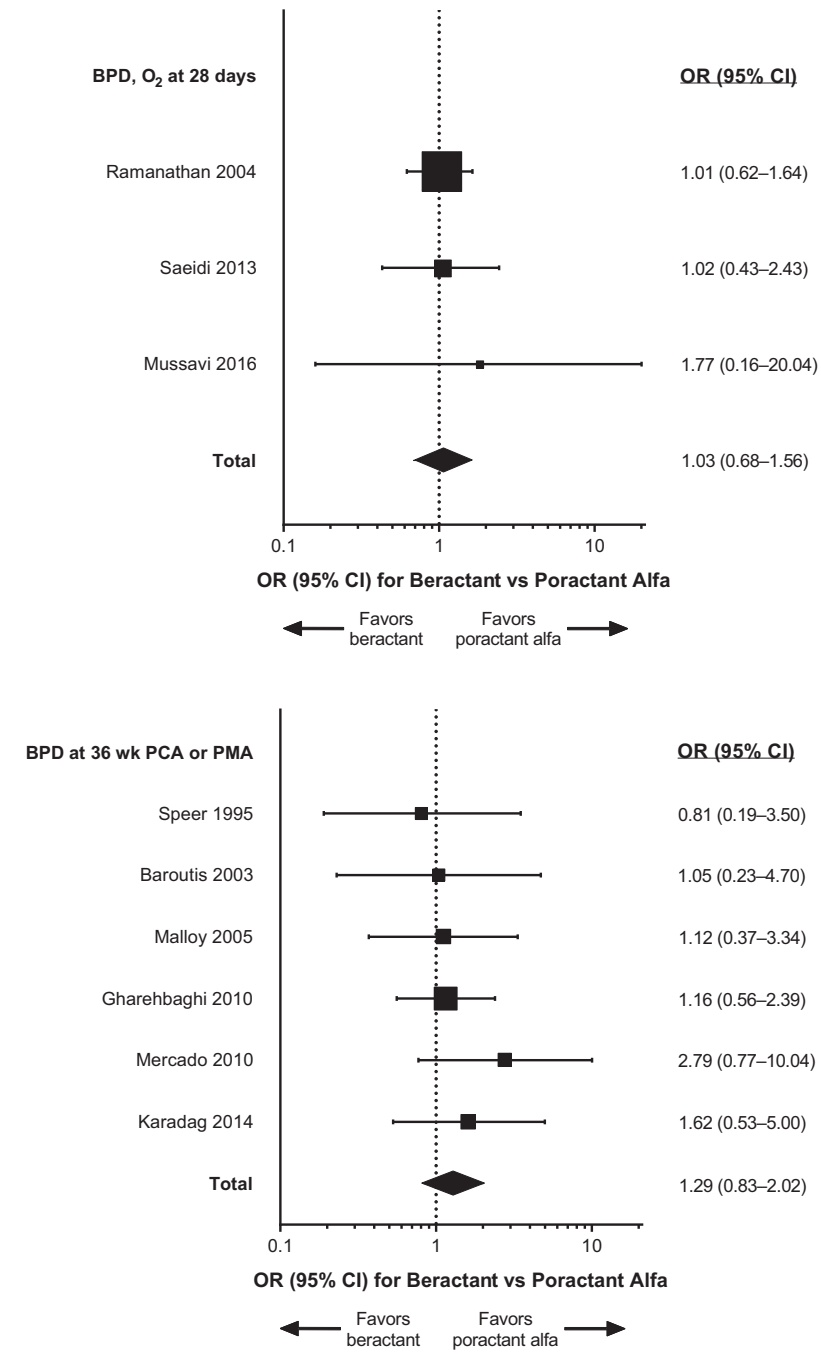

Fig. 6 Forest plots BPD from sensitivity analyses that included only data from RCTs in which BPD defined as the need for oxygen at day 28 after birth or evaluated at 36 weeks PCA or PMA. BPD bronchopulmonary dysplasia, CI confidence interval, OR odds ratio, PCA postconceptional age, PMA postmenstrual age, RCT randomized controlled trial.

contributed a total of 816 patients to the pooled metaanalyses of treatment with beractant or poractant alfa, comprised over half of the 13 RCTs that we included for meta-analysis of the risk of death, and were on average more recent (published in 2010-2018) compared with the studies analyzed by Singh et al. (1995-2014) or Zhang et al. (1995-2013) [9]. Six studies were included in all three works (and in meta-analyses in each case, except for data from Fujii et al. in our work) [19, 21, 23, 25, 28, 30], two were included in the present article and by Singh et al. (and in meta-analyses) [27, 29], and two were included in the present article (not subjected to meta-analysis by us because they were RWE studies) and by Zhang et al. [15, 16]. Singh et al. included one article, a doctoral dissertation about a small study [35], which we did not because it was not published in a peer-reviewed format. Thus, the metaanalysis results reported here are founded upon a sample that was more robust and also likelier to reflect current clinical practice compared with the previous meta-analysis publications. A final aspect that might have influenced the findings in our meta-analyses as compared with prior publications was the choice, when similar endpoints were reported (e.g., death at 28 days after birth or at 36 weeks PCA, as in Ramanathan et al. 2004 [19]), of which one to subject to meta-analysis. We chose the former outcome (death at 28 days) because it appeared more applicable across the studies available for pooling.

Despite the increased power to observe treatment differences that is afforded by comparing studies or pooling multiple trials in a meta-analysis, any such efforts have limitations. For the RWE studies, the limitation of study heterogeneity was substantial enough that meta-analysis was not attempted. For example, in the studies analyzed here, the follow-up period was often vague or differed between studies. Normally, studies follow up only as long as patients are in the neonatal intensive care unit (NICU), where patients typically reside for about 1 month. BPD, pneumothorax, and ALS are usually observed within a 1-month timeframe. Any deaths during the period are noted. Patients who survive their first month typically leave the NICU. The studies also had variable inclusion criteria that were reflected in the characteristics of the study populations; for example, there were large differences in gestational age cutoffs and, as a result, birth weights. The definitions of outcomes were frequently not uniform; however, this reflects the reality that clinical practices are themselves diverse. Some outcomes (e.g., ALS) were sporadically reported, reducing the power to detect differences between treatment groups. Doses of surfactant did not always match the US product label recommendations, although the similar findings among the meta-analyses that included all doses and the sensitivity analyses that included only doses that matched the US product label argue against a prominent effect, and off-label dosing also reflects clinical practice. Also, with regards to death, BPD, and pneumothorax, the ORs and corresponding $95 \%$ CIs for beractant $100 \mathrm{mg} / \mathrm{kg}$ vs. poractant alfa $100 \mathrm{mg} / \mathrm{kg}$ in our analyses were 1.39 (0.87-2.22), $0.99 \quad(0.64-1.52)$, and 1.24 (0.64-2.41), respectively, emphasizing the similar efficacy of both products. However, the studies comparing beractant $100 \mathrm{mg} / \mathrm{kg}$ with poractant alfa $100 \mathrm{mg} / \mathrm{kg}$, although they did not demonstrate significant differences between treatments in these sensitivity analyses, were largely limited to research that was older or conducted under circumstances in which the dose regimen may have been chosen due to limited resources. Current practice is dominated by administration of poractant alfa at its recommended initial dose $(200 \mathrm{mg} / \mathrm{kg} ; 2.5 \mathrm{~mL} / \mathrm{kg})$ rather than at a lower dose 
$(100 \mathrm{mg} / \mathrm{kg} ; 1.25 \mathrm{~mL} / \mathrm{kg})$, and so with a volume by patient weight that is similar to that for beractant $100 \mathrm{mg} / \mathrm{kg}$ $(4 \mathrm{~mL} / \mathrm{kg})$. Although a slightly larger volume is used with beractant, the amount of total phospholipid received is consistent with the normal content of phospholipid in the natural surfactant produced by at-term infants [36]. When $100 \mathrm{mg}$ of phospholipid is used with poractant alfa, even though the amount of phospholipid is similar to physiologic conditions, the clinical outcome does not appear to be similar to beractant, at least with regards to the incidence of death. This might be due to a smaller volume of distribution (1.25 vs. $4 \mathrm{~mL}$ ). Although there is a larger exposure to phospholipids than in physiologic conditions, when the volume of poractant alfa is increased to $2.5 \mathrm{mg} / \mathrm{kg}$ (i.e., $200 \mathrm{mg} / \mathrm{kg}$ ), there is an evident improvement in clinical outcomes (in particular with incidence of death), and is thus comparable to beractant. All sensitivity analyses were limited by smaller sample sizes relative to the primary analyses, with the result that an absence of significance could have reflected the lesser statistical power. A final limitation is that, for RCTs of surfactants, full blinding is generally impossible because of different volumes and methods of administration among products, which might have resulted in bias among study personnel. Regardless of these potential limitations, the consistency of the findings supports the overall validity of the findings. Methods of less invasive surfactant administration might improve some outcomes as they become more widely adopted [37-39].

The systematic review of RWE studies and our primary meta-analysis of RCTs indicate that the incidences of mortality, BPD, pneumothorax [40], and ALS for beractant compared with poractant alfa were similar in premature infants treated for acute RDS. The results of our primary meta-analysis of RCTs was in agreement with that reported by Zhang et al. [10], which only included death as an outcome, and with Singh et al. [9], for all the aforementioned outcomes, except for risk of death. Although there are inherent limitations to RWE studies (namely study heterogeneity, greater heterogeneity of the RWE populations due to lack of randomization, and variation in the definitions of outcomes), RWE studies are more representative of and more accurately reflect the experiences of patients in usual clinical practice. In the RWE studies, no significant differences in outcomes were observed between beractant and poractant alfa, suggesting that there may be no meaningful real-world differences between these treatments.

Acknowledgements The authors thank AbbVie employees, Damian Gruca, MD, for designing the literature search, and Sherry Kim for her assistance with its implementation. Michael J. Theisen, Ph.D., and Janet E. Matsuura, Ph.D., of Complete Publication Solutions, LLC (North Wales, PA, USA), a CHC Group company, provided medical writing and editorial support to the authors in the development of the manuscript, which was funded by AbbVie. AbbVie funded the work, contributed to its design, and was involved in the collection, analysis, and interpretation of the data and in the writing, review, and approval of the publication.

\section{Compliance with ethical standards}

Conflict of interest MSL received travel and lectures grants from AbbVie Inc. and served as a consultant for Chiesi and AbbVie. CRN has no relevant conflicts of interest. $\mathrm{PB}, \mathrm{KU}$, and MM-T are employees of AbbVie and may own AbbVie stock and/or stock options.

Publisher's note Springer Nature remains neutral with regard to jurisdictional claims in published maps and institutional affiliations.

Open Access This article is licensed under a Creative Commons Attribution 4.0 International License, which permits use, sharing, adaptation, distribution and reproduction in any medium or format, as long as you give appropriate credit to the original author(s) and the source, provide a link to the Creative Commons license, and indicate if changes were made. The images or other third party material in this article are included in the article's Creative Commons license, unless indicated otherwise in a credit line to the material. If material is not included in the article's Creative Commons license and your intended use is not permitted by statutory regulation or exceeds the permitted use, you will need to obtain permission directly from the copyright holder. To view a copy of this license, visit http://creativecommons. org/licenses/by/4.0/.

\section{References}

1. Sweet DG, Carnielli V, Greisen G, Hallman M, Ozek E, Plavka R, et al. European consensus guidelines on the management of respiratory distress syndrome-2016 update. Neonatology. 2017; 111:107-25.

2. Gallacher DJ, Hart K, Kotecha S. Common respiratory conditions of the newborn. Breathe (Sheff). 2016;12:30-42.

3. Polin RA, Carlo WA, Committee on Fetus and Newborn, American Academy of Pediatrics. Surfactant replacement therapy for preterm and term neonates with respiratory distress. Pediatrics. 2014;133:156-63.

4. Taylor G, Jackson W, Hornik CP, Koss A, Mantena S, Homsley $\mathrm{K}$, et al. Surfactant administration in preterm infants: drug development opportunities. J Pediatr. 2019;208:163-8.

5. Survanta (beractant). Full prescribing information. North Chicago, IL: AbbVie Inc.; 2012.

6. Curosurf (poractant alfa). Full prescribing information. Parma, Italy: Chiesi Farmaceutici, S.p.A.; 2014.

7. Survanta (beractant). Summary of product characteristics. Maidenhead, UK: AbbVie Ltd.; 2015.

8. Curosurf (poractant). Summary of product characteristics. Manchester, UK: Chiesi Limited; 2018.

9. Singh N, Halliday HL, Stevens TP, Suresh G, Soll R, Rojas-Reyes MX. Comparison of animal-derived surfactants for the prevention and treatment of respiratory distress syndrome in preterm infants. Cochrane Database Syst Rev. 2015;21:CD010249.

10. Zhang L, Cao HY, Zhao S, Yuan LJ, Han D, Jiang H, et al. Effect of exogenous pulmonary surfactants on mortality rate in neonatal respiratory distress syndrome: a network meta-analysis of randomized controlled trials. Pulm Pharmacol Ther. 2015;34:46-54.

11. Sherman RE, Anderson SA, Dal Pan GJ, Gray GW, Gross T, Hunter NL, et al. Real-world evidence-what is it and what can it tell us? N Engl J Med. 2016;375:2293-7. 
12. US Food and Drug Administration. Framework for FDA's realworld evidence program. Silver Spring, MD: US Food and Drug Administration; 2018.

13. Guyatt G, Oxman AD, Akl EA, Kunz R, Vist G, Brozek J, et al. GRADE guidelines: 1. Introduction-GRADE evidence profiles and summary of findings tables. J Clin Epidemiol. 2011;64: 383-94.

14. National Collaborating Centre for Mental Health. Drug misuse: psychosocial interventions. NICE Clinical Practice Guidelines No. 51. Leicester, UK: The British Psychological Society \& The Royal College of Psychiatrists; 2008.

15. Trembath A, Hornik CP, Clark R, Smith PB, Daniels J, Laughon $\mathrm{M}$, et al. Comparative effectiveness of surfactant preparations in premature infants. J Pediatr. 2013;163:955-60. e951.

16. Ramanathan R, Bhatia JJ, Sekar K, Ernst FR. Mortality in preterm infants with respiratory distress syndrome treated with poractant alfa, calfactant or beractant: a retrospective study. J Perinatol. 2013;33:119-25.

17. Paul S, Rao S, Kohan R, McMichael J, French N, Zhang G, et al. Poractant alfa versus beractant for respiratory distress syndrome in preterm infants: a retrospective cohort study. J Paediatr Child Health. 2013;49:839-44.

18. Naseh A, Yekta BG. INSURE method (INtubation-SURfactantExtubation) in early and late premature neonates with respiratory distress: factors affecting the outcome and survival rate. Turk $\mathbf{J}$ Pediatr. 2014;56:232-7.

19. Ramanathan R, Rasmussen MR, Gerstmann DR, Finer N, Sekar K, North American Study Group. A randomized, multicenter masked comparison trial of poractant alfa (Curosurf) versus beractant (Survanta) in the treatment of respiratory distress syndrome in preterm infants. Am J Perinatol. 2004;21:109-19.

20. Eras Z, Dizdar EA, Kanmaz G, Guzoglu N, Aksoy HT, Altunkaya $\mathrm{GB}$, et al. Neurodevelopmental outcomes of very low birth weight preterm infants treated with poractant alfa versus beractant for respiratory distress syndrome. Am J Perinatol. 2014;31:463-8.

21. Fujii AM, Patel SM, Allen R, Doros G, Guo CY, Testa S. Poractant alfa and beractant treatment of very premature infants with respiratory distress syndrome. J Perinatol. 2010;30:665-70.

22. Mussavi M, Mirnia K, Asadollahi K. Comparison of the efficacy of three natural surfactants (Curosurf, Survanta, and Alveofact) in the treatment of respiratory distress syndrome among neonates: a randomized controlled trial. Iran J Pediatr. 2016;26:e5743.

23. Gharehbaghi MM, Sakha SH, Ghojazadeh M, Firoozi F. Complications among premature neonates treated with beractant and poractant alfa. Indian J Pediatr. 2010;77:751-4.

24. Mirzarahimi M, Barak M. Comparison efficacy of Curosurf and Survanta in preterm infants with respiratory distress syndrome. Pak J Pharm Sci. 2018;31:469-72.

25. Dizdar EA, Sari FN, Aydemir C, Oguz SS, Erdeve O, Uras N, et al. A randomized, controlled trial of poractant alfa versus beractant in the treatment of preterm infants with respiratory distress syndrome. Am J Perinatol. 2012;29:95-100.

26. Najafian B, Karimi-Sari H, Khosravi MH, Nikjoo N, Amin S, Shohrati M. Comparison of efficacy and safety of two available natural surfactants in Iran, Curosurf and Survanta in treatment of neonatal respiratory distress syndrome: a randomized clinical trial. Contemp Clin Trials Commun. 2016;3:55-9.

27. Karadag N, Dilli D, Zenciroglu A, Aydin B, Beken S, Okumus N. Perfusion index variability in preterm infants treated with two different natural surfactants for respiratory distress syndrome. Am J Perinatol. 2014;31:1015-22.

28. Baroutis G, Kaleyias J, Liarou T, Papathoma E, Hatzistamatiou Z, Costalos C. Comparison of three treatment regimens of natural surfactant preparations in neonatal respiratory distress syndrome. Eur J Pediatr. 2003;162:476-80.

29. Malloy CA, Nicoski P, Muraskas JK. A randomized trial comparing beractant and poractant treatment in neonatal respiratory distress syndrome. Acta Paediatr. 2005;94:779-84.

30. Speer CP, Gefeller O, Groneck P, Laufkotter E, Roll C, Hanssler $\mathrm{L}$, et al. Randomised clinical trial of two treatment regimens of natural surfactant preparations in neonatal respiratory distress syndrome. Arch Dis Child Fetal Neonatal Ed. 1995;72:F8-13.

31. Mercado VV, Cristea I, Ali N, Pham CC, Buescher E, Yang J, et al. Does surfactant type cause a differential proinflammatory response in preterm infants with respiratory distress syndrome? Adv Ther. 2010;27:476-82.

32. Saeidi R, Hamedi A, Javadi A, Gholami Robatsangi M, Dinparvar SK. Comparison of side effect of Survanta and Curosurf in decreasing mortality due to respiratory distress syndrome (RDS) in premature infants admitted in NICU of Ghaem Hospital on 2006-2008. Iran J Neonatol. 2013;4:7-12.

33. Terek D, Gonulal D, Koroglu OA, Yalaz M, Akisu M, Kultursay N. Effects of two different exogenous surfactant preparations on serial peripheral perfusion index and tissue carbon monoxide measurements in preterm infants with severe respiratory distress syndrome. Pediatr Neonatol. 2015;56:248-55.

34. Tridente A, De Martino L, De Luca D. Porcine vs bovine surfactant therapy for preterm neonates with RDS: systematic review with biological plausibility and pragmatic meta-analysis of respiratory outcomes. Respir Res. 2019;20:28.

35. Halahakoon WL. A study of cerebral function following surfactant treatment for respiratory distress syndrome. Doctoral dissertation. United Kingdom: Queen's University of Belfast; 1999.

36. Jobe AH. Mechanisms to explain surfactant responses. Biol Neonate. 2006;89:298-302.

37. Dumpa V, Bhandari V. Surfactant, steroids and non-invasive ventilation in the prevention of BPD. Semin Perinatol. 2018; 42:444-52.

38. Härtel C, Paul P, Hanke K, Humberg A, Kribs A, Mehler K, et al. Less invasive surfactant administration and complications of preterm birth. Sci Rep. 2018;8:8333.

39. Langhammer K, Roth B, Kribs A, Göpel W, Kuntz L, Miedaner F. Treatment and outcome data of very low birth weight infants treated with less invasive surfactant administration in comparison to intubation and mechanical ventilation in the clinical setting of a cross-sectional observational multicenter study. Eur J Pediatr. 2018;177:1207-17.

40. Brown S, Hurren J, Sartori H. Poractant alfa versus beractant for neonatal respiratory distress syndrome: a retrospective cost analysis. J Pediatr Pharm Ther. 2018;23:367-71. 